\title{
Safety Locking System of Car Door Using Sensors
}

\author{
Venkatesh PL, Vivek C \\ Department of Mechanical Engineering, RMK Engineering College, Chennai, India \\ Department of Mechanical Engineering, RMK Engineering College, Chennai, India
}

\begin{abstract}
The safety locking system is designed to prevent accidents happening while opening the car door and to provide safety to passengers and pedestrians. To prevent accident due to passenger's carelessness, in this planned to control the car door locking automatically using distance measuring system, child lock and ultrasonic sensors. Due to this passengers can't open their car door from inside while object is moving towards the car. The entire system is controlled with the help of advanced microcontroller. In this proposed system it will be fitted with a distance measuring ultrasonic sensor in car rear view mirror on both sides. This sensor sends and receives the signal near the outer surface of the car door. Due to this if any object approaches the car, the signal get reflect and received by the receiver. Due to quick return of the signal, the microcontroller sends signal to the child lock and it get activates according to the program performed on the microcontroller. Due to this the passengers can't open the car from inside, so sudden opening can be prevented. But we can open the car from outside. If the object/person moved then the signal passes long distance and the receiving time get increased, so the controller sends the signal to the lock and it comes back to its original position.
\end{abstract}

Keywords: ultrasonic sensor, child lock, distance measuring system, car door accidents

\section{Introduction}

The cars are nowadays designed with the monitoring system which could measure the distance between the two objects form few centimetres to a few metre. The distance between the vehicles are generally calculated using time of flight technique. It is used to warn the driver to avoid accident [1]. But warning systems are not much effective hence in this approach the occurrence of accident is eliminated as early as possible. To achieve such a system the sensors are preferred which is having timely manner action. The electronic system with fast acting microcontroller performs this function. Sensors are defined as device (or transducer) that transform physical quantities such as pressure or acceleration into output signal (usually electrical) that serve as input for control systems [5]. As a prototype the car door with the child lock system will be used to demonstrate the safety locking system to be designed.

\section{Problem}

With the expansion of road facilities, motorization and urbanisation of the country, the number of road accidents have surged. Road traffic injuries (RTI) and fatalities have emerged as a major public health concern, with RTIs having becoming one of the leading causes of deaths, disabilities and hospitalizations which impose severe socio-economic costs across the world.World Health statistics 2008cited in global report on rod safety states that the

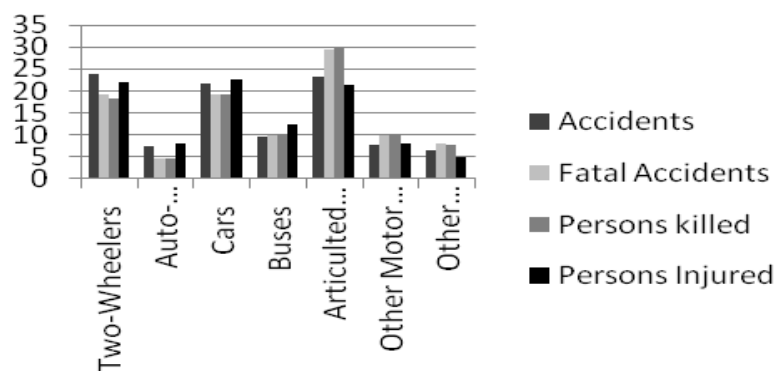

Figure 1: Accident data of different vehicle in 2010
RTIs in 2004 were the $9^{\text {th }}$ leading cause of death and at current rates by 2030 are expected to be the $5^{\text {th }}$ leading cause of death, overtaking HIV/AIDS [14].

During the year 2010, there were around 5 lakh road accidents, which resulted in deaths of 134,513 people and injured more than 5 lakh persons in India. These numbers translate into 1 road accident per minute and 1 road accident death every four minute. Now India is leading china in number of road accidents. The road safety is an issue of national concern, considering its magnitude and gravity and the consequent negative impacts on the economy, public health and the general welfare of the people [13].

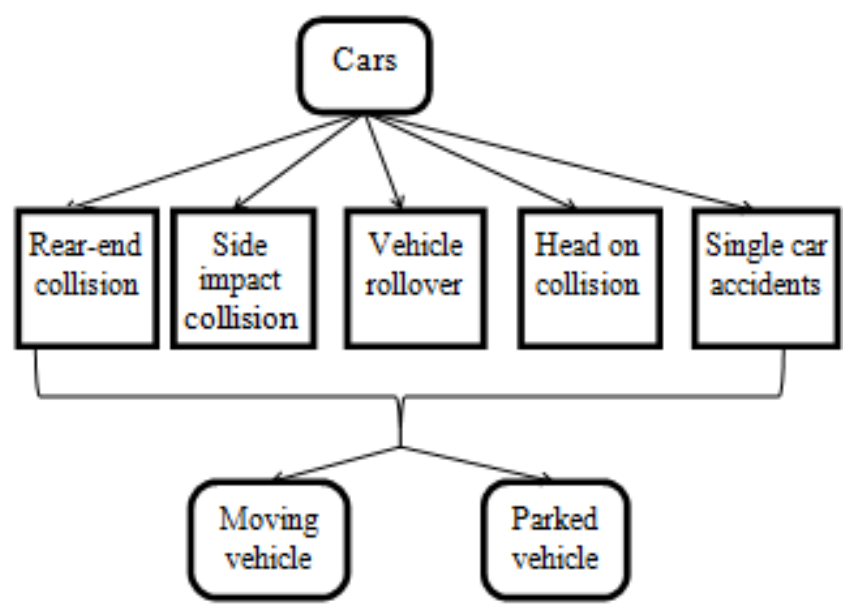

Figure 2: Accidents occurring due to cars

Of the total accidents occurred more accidents occurring on the two-wheelers and cars after heavy duty vehicles. Here this project is concentrating on these categories. Accidents occurring in the cars are of different types. They are as in the above chart.

As mentioned, the accidents occurring in both the moving and parked conditions but more safety measures are taken only during the moving condition. Hence this project concentrates on the accident occurring on the parked vehicle. 


\section{International Journal of Science and Research (IJSR) \\ ISSN (Online): 2319-7064 \\ Index Copernicus Value (2013): 6.14 | Impact Factor (2014): 5.611}

The accident taken into the consideration was car door accidents. Car door accident is one of the accidents occurring in the parked condition. Car door accident is the one which occurs when the passenger or driver carelessly opens the door without the conscious of vehicle or other objects in the door zone [12]. The door zone is the radial rotational distance of $1.2 \mathrm{~m}$ provided to avoid the accident in the car door.The car door accidents are increasing nowadays with the increase in number of cars. Even the percentage of car door accidents or the number of car door accidents to the number of cars is reduced, total number of car door accidents increasing every year [16].

The motorist and cyclist met most number of accidents in the car. The $35 \%$ of accidents occur in the car doors. The motor vehicle occupants and non-occupants killed and injured reveals most are due to pedestrian cars. The accidental opening of door due to carelessness leads to such an accident [16]. Even the well developed countries which are providing door zone in the road also have considerable amount of accident due to opening doors. In developing countries these accidents are increasing now due to the more number of usages of cars in recent days, the absence of door zone and the less awareness about the car door accidents. There is a need for control measures to avoid this type of accident rather than to reduce these types of accidents.

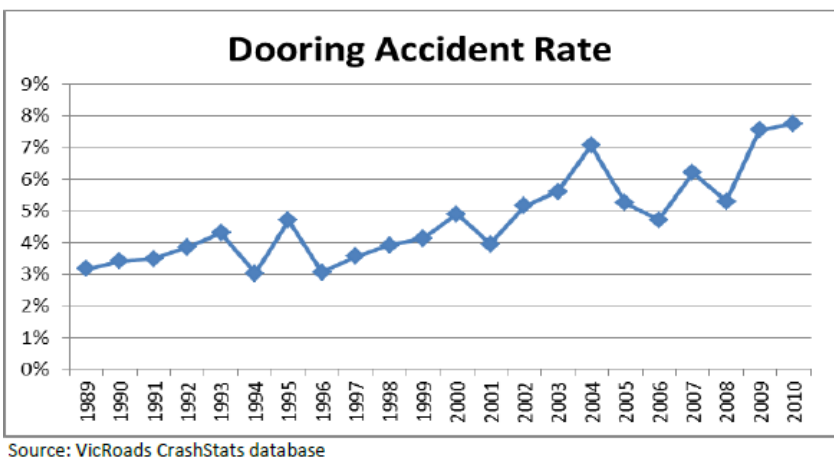

Figure 3: Dooring Accident Rate

In most cases car dooring does not directly results in fatal accidents but fall into the nearby vehicle passing by and got hit by the vehicle after hit by the car doors. Even law/ enforcement are already available in-order to minimise these accidents these measures are not sufficient enough [16]. Hence I suggest a new system to control the car door accidents at its source.

\section{A. Objective of the System}

- The aim of the project is to prevent the car door accidents by a locking system which consists of child lock system and distance measuring ultrasonic sensor.

- To select suitable ultrasonic sensor and to fabricate a long range distance measuring module.

\section{Related Work}

The main problems identified in the existing system and other systems which are implacable are mainly as follows [3]:

- The sophisticated devices such as radar and video camera are very useful but are costlier to implement.
- The warning system with the indication of LED and buzzer are not effective since it cannot be effectively perceived by the drivers during extreme circumstances.

\section{Safety Locking System}

The safety locking system in the suggested system contains the three main systems in it. They are ultrasonic sensor, distance measuring system and child safety lock. As the distance measuring system utilises the ultrasonic sensor for the measurement it is used to identify not only the distance and also the speed of the approaching vehicle. After the calculation of speed and distance of the approaching vehicle, it is thus compared with the preset conditions of speeddistance for which the lock has to be activated. According to the condition made, the locking system operates.

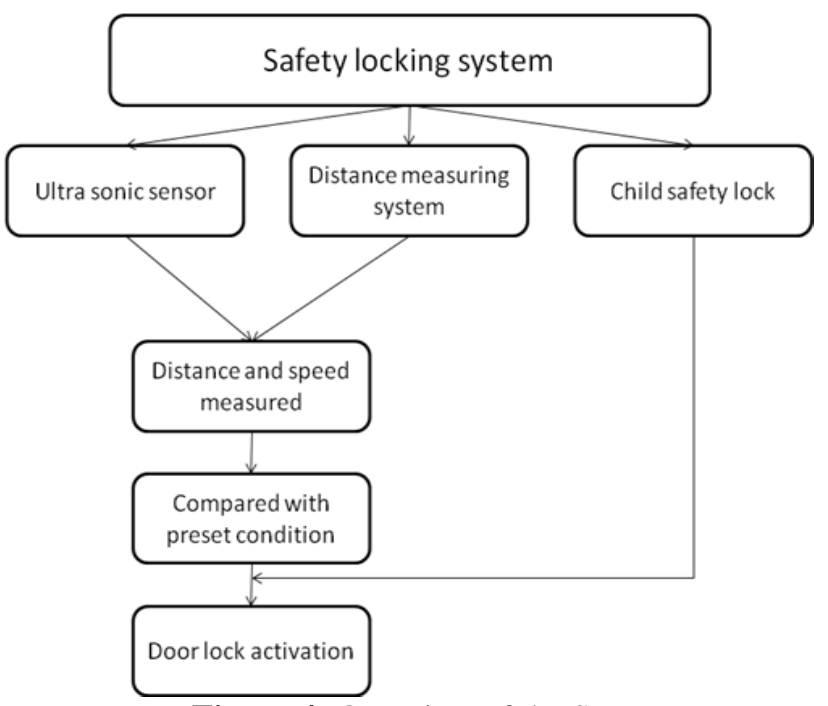

Figure 4: Overview of the System

\subsection{Ultrasonic Distance Measurement}

There are several ways to measure distance without contact. One way is to use ultrasonic waves at $40 \mathrm{kHz}$ for distance measurement [10]. Ultrasonic transducers measure the amount of time taken for a pulse of sound to travel to a particular surface and return as the reflected echo [11]. This circuit calculates the distance based on the speed of sound at $25^{\circ} \mathrm{C}$ ambient temperature and shows it on a 7-segment display. For this, the components required are AT89C2051 microcontroller, $40 \mathrm{kHz}$ ultrasonic transducers, current buffer ULN2003, operational amplifier LM324, four 7-segment displays, five transistors and other components[9]. The ultrasonic transmitter-receiver pair is shown in ultrasonic generators use quartz crystal. In this circuit, a $40 \mathrm{kHz}$ transducer is used. The velocity of sound in the air is $330 \mathrm{~m} / \mathrm{s}$ at $0^{\circ} \mathrm{C}$ and varies with the temperature. In this project, the ultrasonic transmitter unit with a $40 \mathrm{kHz}$ pulse is excited to measure.

The transmitted burst, which lasts for a period of approximately $0.5 \mathrm{~ms}$,it travels to the object in the air and the echo signal is picked up by another ultrasonic transducer unit (receiver), also a $40 \mathrm{kHz}$ pre-tuned unit. The received signal which is very weak is amplified several times in the receiver circuit. Weak echoes also occur due to the signals being directly received through the side lobes. These are ignored as 


\section{International Journal of Science and Research (IJSR) \\ ISSN (Online): 2319-7064 \\ Index Copernicus Value (2013): 6.14 | Impact Factor (2014): 5.611}

the real echo received alone would give the correct distance. Of course, the signal gets weaker if the target is far and will need a higher pulse excitation voltage or a better transducer [15]. Here the microcontroller is used to generate $40 \mathrm{kHz}$ sound pulses. It reads when the echo arrives; it finds the time taken in microseconds for to-and-fro travel of sound waves. Using velocity of $333 \mathrm{~m} / \mathrm{s}$, it does the calculations and shows on the four 7-segment displays the distance in centimetre and millimetres (three digits for centimetre and one or millimetres).

\subsubsection{Ultrasonic Sensor}

Ultrasonic sonar sensors actively transmit acoustic waves and receive them later. This is done by ultrasonic transducers, which transform an electrical signal into an ultrasonic wave and vice versa. The ultrasound signal carries the information about the variables to be measured. The task for the ultrasonic sensors is not merely to detect ultrasound, as intelligent sensors they have to extract the information carried by the ultrasonic signals efficiently and with high accuracy. To achieve this performance, the signals are processed, demodulated and evaluated by dedicated hardware. Algorithms based on models for the ultrasonic signal propagation and the interaction between the physical or chemical variables of interest are employed (Munich, 1994). Furthermore, techniques of a sensor specific signal evaluation are being applied. Ultrasonic sensors can be embedded into a control system that has accesses to the additional sensors, combines information of the different sensors, handles the bus protocols and initiates actions.

An ultrasonic transducer is a device that converts energy into ultrasound, or sound waves above the normal range of hearing for humans (about $20 \mathrm{kHz}$ ). Ultrasonic transducers are widely used in a variety of applications and in technological areas with significant differences in performance characteristics, operating environment, specifications, and actions.

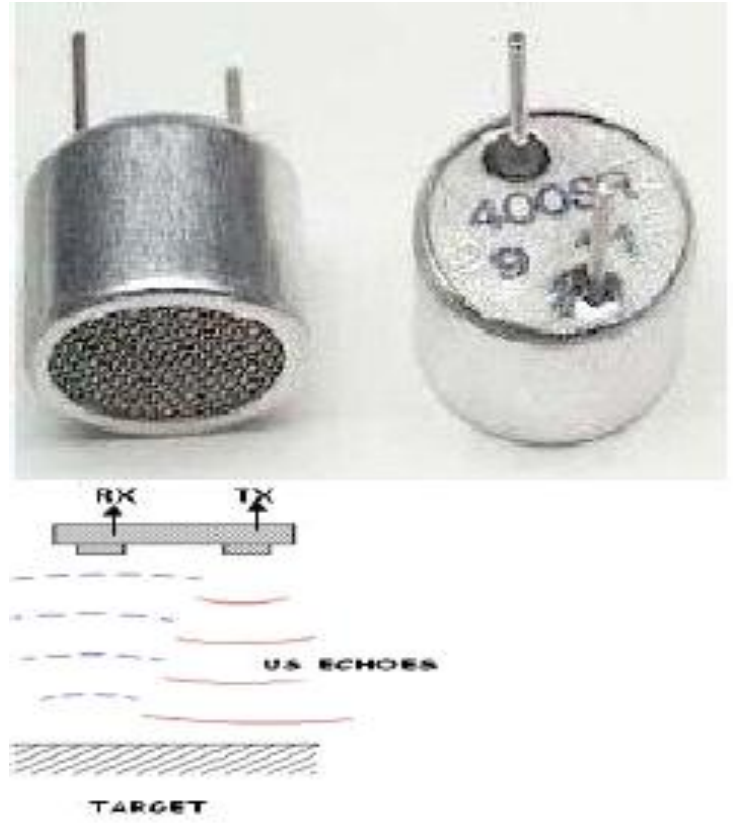

Figure 5: Ultrasonic Transducer (Transmitter and Receiver)
Ultrasonic instrumentation and measurement systems can be found in applications varying from underwater or industrial systems, like finding flaws in materials, to medical imaging, burglar alarms, non-destructive evaluation, humidifiers, sonar and medical ultrasonography [17]. By all this application, this ultrasonic technology is suitable for ultrasonic cleaners (like lenses or other optical parts, and jewellery cleaners), sonochemistry, carrying audio messages, sonic weaponry and robotics. A common use of ultrasonic or ultrasound is in range finding, this use is also called sonar. This works similarly to radar. An ultrasonic pulse is generated in a particular direction. If there is an object in the path of this pulse, part or all of the pulse will be reflected back to the sender as an echo and can be detected. By measuring the difference in time between the pulse being transmitted and the echo being received, it is possible to determine how far away the object is.

\subsubsection{Time of flight measurement}

The most common method of distance measurement is based on time elapsed between transmission and reception of an ultrasonic pulse called time-of flight (ToF) method. The duration of echo and amplitude are used to analyse the distance $\mathrm{x}$ and the characteristics of the object.

$$
\mathrm{x}=\mathrm{vt} / 2
$$

where $\mathrm{t}=$ time duration of the burst.

\subsection{Child Safety Lock}

Child safety locks are built into the rear doors of most cars to prevent rear seat passengers from opening the doors both during transit and while the vehicle is stationary; vehicles have been built with this feature since the early 1980s.

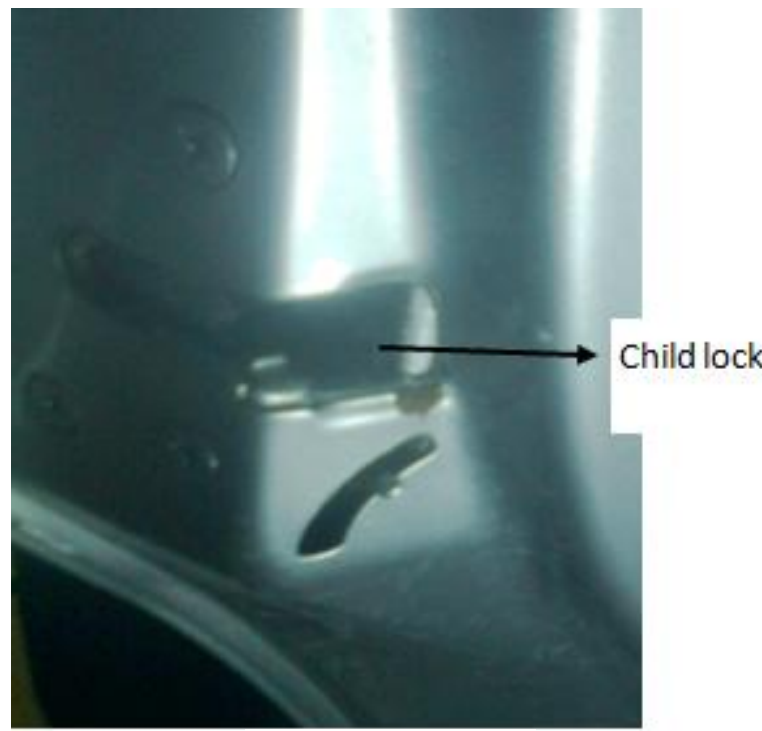

Figure 6: Child lock

They provide the vehicle driver with a simple, safe \& secure method to prevent exit from the car. Although called a child lock it is equally effective for adult passengers. In this state the passenger cannot open the door from the inside and is effectively locked in, the passenger can only be released by someone lifting the outside handle 


\section{International Journal of Science and Research (IJSR) \\ ISSN (Online): 2319-7064 \\ Index Copernicus Value (2013): 6.14 | Impact Factor (2014): 5.611}

\section{System Description}

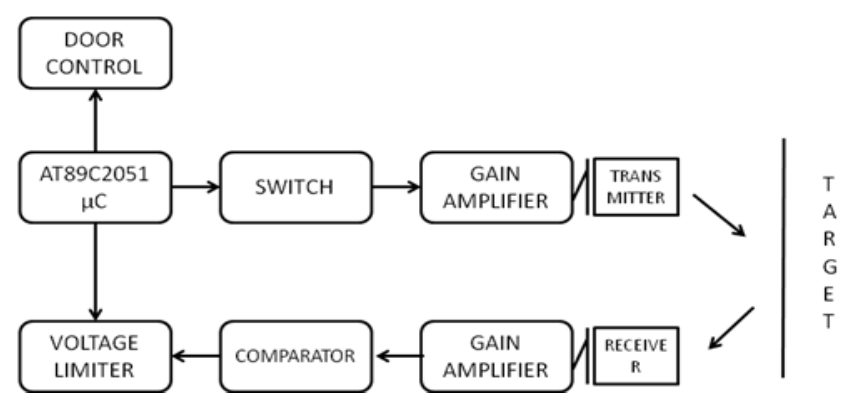

Figure 7: Block diagram of safety locking system

This is the block diagram of the safety locking system which contains the distance measuring system with the ultrasonic sensor and provides control to the door lock while the condition made on the microcontroller is achieved.

\subsection{Microcontroller}

The microcontroller AT89C2051 is chosen for the distance measurement. The microcontroller itself contains analog comparator in it which is more useful in comparing the preset condition with the distance actually measured with the measuring system. It works on the principle that the latching mechanism is operated only when object is detected for two times. The latch will not operate on its first time of detection since it may mislead that the vehicle coming on the other lane may be detected.

\subsection{Door Control}

The signal will be sent to the lock through the body control module. Body control module is the central system which is used for sending signal to different doors and windows. The microcontroller will send the signal to body control and then it sends to the corresponding doors.

\section{Software}

The software is written in Assembly language and assembled using 8051 cross-assembler. It is well commented and easy to understand. The pulse train for $0.5 \mathrm{~ms}$ is started by making pin 8 high and low alternately for 12.5 microseconds so that the pulse frequency is $40 \mathrm{kHz}$. After 25 such pulses have passed, a waiting time is given to avoid direct echoes for about $20 \mu \mathrm{s}$. Then the signal is awaited, while the timer runs counting time in microseconds. When the echo arrives, port3 pin P3.6 goes high, the timer reads and the 16-bit number is divided by twice the velocity and converted into decimal format as a 4-digit number. If the echo does not arrive even after waiting period, the waiting loop is broken and the pulse train sequence is started once again. If the echo comes within this time, it is displayed for a half second proceeding to another measurement. Thus, the display appears continuous and flicker-free. This is used to evaluate the ultrasonic sensor measuring range.

\section{Conclusion}

At this stage, the components for fabricating the safety locking system were procured and the software for distance measurement is being developed. When the ultrasonic sensor senses up to the desired distance the program for the latching operation of child lock developed and the system would be checked for its proper function. The system will be improved with its performance, when the sensing range is improved. When the fabrication of the safety locking system is developed, the system is coordinated with the body control module and the system is come up with the priorities for different types of action.

\section{References}

[1] Faisal Muhaiyuddin, RahmatSanudin, Mohd Helmy Abd Wahab and Ayob Johari, "Development of Digital Vehicle Distance Monitoring system", (2009) IEEE, 612-616.

[2] Dai Qiang Wang, Shi You Gao, Yu Qing Chen, Yi Wang and Qiao Liu, "Intelligent control system based on CAN Bus for Car Doors and windows", (2008).

[3] Fred Yu, Bozena Kaminska and PawelGburzynski, "A New Driving Assistant for Automobiles”, (2007) IEEE 1199-1202.

[4] Thomas M. Galla, Dietmar Schreiner, Wolfgang Forster, Christof Kutschera, Karl M. Goschka, and Martin Horauer. "Refactoring an Automotive Embedded Software Stack using the Component Based Paradigm", (2005).

[5] Bill Fleming, “Automotive electronics”, IEEE vehicular technology magazine, September 2006, 49-52.

[6] Jaroslaw Majchrzak, Mateusa Michalski and Grzegorz Wiczynski, "Distance Estimation With a Long-Range Ultrasonic Sensor System", (2009), IEEE, 767-773.

[7] Kenneth J. Ayala, The 8051 Microcontroller Architecture, Programming\& Applications, West Publishing Company, College \& School Division,1996.

[8] Muhammed Ali Mazidi, Janice Gillispie Mazidi, "The 8051microcontroller \& Embedded Systems”, Pearson Education.

[9] Datasheet of components (AT89C2051, ULN20003, CD4049, LM324, 1N4148, 1N4007, voltage regulator)

[10] T.K. Bhaumik, D. Sarkar “A Microcontroller Based Ultrasonic Range Finding Module for target detection from a Mobile Robot"

[11] A.K. Shrivastava "Distance measurement of an object or obstacle by ultrasonic sensors using P89C51RD2", 6468, Feb (2010).

[12]Door zone cited at http://en.wikipedia.org/wiki/door zone.

[13] "Road Accidents in India 2010" by Government of India and Ministry of Road Transport and Highways

[14] "World Report on Road Traffic Injury Prevention", by World Health Organisation

[15] Ajay Kumar Shrivastava, "Effect of variation of separation between the ultrasonic transmitter and receiver on the accuracy of distance measurement", 1924, International journal of computer science and information technology, Nov (2009)

[16] Greg barber, "Legislative council standing committee on Economy and Infrastructure

[17] Sangwon Lee, Dukhee Yoon and AmitabhaGhosh, "Intelligent Parking Lot Application Using Wireless Sensor Networks", (2008), IEEE, 48-57. 


\section{Author Profile}

PL.Venkatesh is in Department of Mechanical Engineering, R. M. K. Engineering College, Anna University, Chennai, India

C.Vivek is in Department of Mechanical Engineering, R. M. K. Engineering College, Anna University, Chennai, India 